\title{
Olfactory Sensitivity Change in the Dysmenorrhea Women: A Cross-Sectional Study
}

\author{
Ren-Jen Hwang ${ }^{1,2,3,}$, , Yu-Jia Yan ${ }^{1}$, Hsin-Ju Chen ${ }^{1}$, Yu-Sheun Lee ${ }^{1,4}$, Yueh-O Chuang ${ }^{2}$ \\ ${ }^{1}$ Department of Nursing, Chang Gung University of Science and Technology, Taoyuan, Taiwan \\ ${ }^{2}$ Department of Nursing, Chang Gung Memorial Hospital, Linkou, Taiwan \\ ${ }^{3}$ Research Center of Clinical Competency Center, Chang Gung University of Science and Technology, Taoyuan, Taiwan \\ ${ }^{4}$ Department of Nursing, China Medical University Hospital, Taichung City, Taiwan
}

Email address:

rjhuang@mail.cgust.edu.tw (Ren-Jen Hwang), goshassault@gmail.com (Yu-Jia Yan), hjchen01@hotmail.com (Hsin-Ju Chen), qoo60824@yahoo.com.tw (Yu-Sheun Lee),j22092@egmh.org.tw (Yueh-O Chuang)

${ }^{*}$ Corresponding author

\section{To cite this article:}

Ren-Jen Hwang, Yu-Jia Yan, Hsin-Ju Chen, Yu-Sheun Lee, Yueh-O Chuang. Olfactory Sensitivity Change in the Dysmenorrhea Women: A Cross-Sectional Study. Journal of Gynecology and Obstetrics. Vol. 6, No. 4, 2018, pp. 86-93. doi: 10.11648/j.jgo.20180604.13

Received: July 31, 2018; Accepted: August 14, 2018; Published: September 3, 2018

\begin{abstract}
Background: Dysmenorrhea is accompanied by abnormal brain metabolism in affective cortical-limbic structures; regions are functionally involved in nociceptive and olfactory processing. The study aims to examine the manifestation of chemical sensitivity changes in women with dysmenorrhea. Methods: A cross-sectional correlation research design was used for this study. Five subsets of odors (lemon, $\mathrm{H}_{2} \mathrm{~S}$, ethyl, acetone, and rose) were tested on 120 women. The visual analogue scale was presented to evaluate the intensity (strong to weak) and hedonic value (pleasant to unpleasant) of each odor. The participants underwent Pain index of dysmenorrhea questionnaire (DQ) and psychophysical assessment after performing olfactory tests. Results: (1) An increased unpleasantness rating of the $\mathrm{H}_{2} \mathrm{~S}$ odorant was associated with the degree of dysmenorrhea. DQ-severity score was the statistical significance of the predictor variable on $\mathrm{H}_{2} \mathrm{~S}$ odorant perception alteration $(P=.006)$, after controlling the collinear variables from regression. (2) Lemon, acetone, and ethyl odorant as positive hedonic values recognized were not significantly related to the DQ pain scores. (3) Additionally, decreased perception of the intensity of the rose odorant was related to rising dysmenorrheal frequency level. $(P=.015)$. (4) Women with higher DQ score were positively related to psychological distress such as anxiety, depression and stress level. Conclusions: Findings disclosed that significantly raised the unpleasantness level of odorant $\mathrm{H}_{2} \mathrm{~S}$, and weakening the intensity value of the pleasantness rose odorant relates to women with severe dysmenorrhea symptoms. The neurobiological bases of olfactory sensitivity changes refer to olfactory-limbic system disturbances and appropriate management of menstrual cramps were discussed.
\end{abstract}

Keywords: Dysmenorrhea, Olfactory Sensitivity, Psychometric Test

\section{Background}

Dysmenorrhea (menstrual cramps) is one of the most common gynecological disorders, affecting almost half the female population of the world. Primary dysmenorrhea (PDM) is menstrual pain without pelvic abnormalities and usually starts one to two years after menarche (the beginning of menstruation). Secondary dysmenorrhea results from a specific underlying disease or disorder. Eighty percent of women experience PDM and suffer from serious cramps that interfere with normal activities. Ongoing menstrual pain is accompanied by abnormal brain metabolism in affective cortical-limbic $[1,2]$, regions that are involved in nociceptive and olfactory processing. According to neuroimaging evidence, the circuitry of the olfactory system is located in the basal limbic forebrain [8]. Dysmenorrhea is still an important public health problem. The long-term effects of dysmenorrhea on odor perception are yet to be evaluated.

Chronic pain is defined as pain that lasts longer than six months. Previous studies have observed significant somatosensory threshold changes and alterations in psychoemotional states in chronic pain patients and menstrual pain 
groups [3-5]. Pain is an inescapable stressor associated with activation of the hypothalamic- pituitary-adrenal (HPA) axis. Recurrent nociceptive stimuli causing neural sensitization or hyperalgesia to heat and pressure stimulation have been reported in dysmenorrhea women [3, 4]. Further investigations have revealed that repeated pain perceptions also triggered the development of heightened arousal to emotionally salient cues, with participants under higher psychological distress persistently re-experiencing traumatic events and displaying an increase in sensory evoked response [5-7]. The mammalian olfactory system detects chemical information from the environment, and a central part decodes the olfactory signal and connected to brain areas. In fact, women suffer menstrual cramps often recognized be a natural biological phenomenon thus adequate manages is disregarded. Olfactory sensitivity and threshold change in dysmenorrheal women is a point of interest in this current preliminary survey.

It is comprehended that the chronic pain is associated with various psychological symptoms or behavioral reactions. Chronic pain is a common problem that is presented in various emotions such as depression and anxiety and it affects the quality of life as it becomes a stressor. Surveys to understand psychological aspects of chronic pain in periodical dysmenorrhea are relatively few before. The Depression and Anxiety inventory has been used for basic and clinical settings to detect and measure the functional manifestations of psychological state for chronic pain client [8-10]. The study was primarily on evaluation of odor perception together with assessed associated psychometric factors according to dysmenorrhea experience. Besides, the elementary relationship of psychological properties and olfactory evaluation were also conducted.

This is the first study to evaluate the impact on the olfactory function in dysmenorrheal women. The most important components of odor perception are the intensity, described as weak or strong, and the hedonic value, described as pleasant (positive) or unpleasant (negative). Previous research has linked the incidence of dysmenorrhea to somatosensory threshold changes, with chronic pain inducing neural sensitization and altering the forebrain morphology $[1,2]$. The present study hypothesizes that odor perception may undergo changes according to women's dysmenorrhea experiences (DE).

\section{Method and Material}

\subsection{Procedure / Design}

A cross-sectional correlation research design was used for this study. Descriptive studies on correlation design are often the first tentative approach to a new event or condition, indicating evidence-based practice [11]. The present study was achieved through convenience and purposive sampling to compare changes in the perception (hedonic value and intensity) of five odors as a consequence of experiencing menstrual pain. We tested five subsets of odors (lemon, $\mathrm{H}_{2} \mathrm{~S}$, ethyl, acetone, rose) on 120 women. 15-ml flasks were filled with 7-8 $\mathrm{ml}$ with five different odorants and presented to the participants. Participants were asked to smell the odorant tube while refraining from tasting its contents or touching the tube with the nose during the test. To ensure the safety of the participants, we presented each odorant vial $1 \mathrm{~cm}$ below the participants' nose then asked them to sniff. The visual analogue scale (VAS) was used and subjects were required to rate the intensity (strong to weak) and hedonic value (pleasant to unpleasant) when the participants were exposed to the 5 odors. The data collection process was completely safe and accompanied by trained nurses through whole course. The screening is completely painless and noninvasive (no needles or x-ray exposure). After performing the olfactory assessments, the participants then underwent the psychometric tests and completed a brief DE questionnaire (DQ).

\subsection{Participants}

We recruited 122 undergraduate female students in Taiwan to participate in this study. Each participant provided informed consent prior to the evaluations. The participants were between 18 and 25 years of age, reported to have a normal sense of smell and were in good respiratory health when tested. Exclusion criteria included broncho-pulmonary or neurological disease, other chronic pain, pregnancy or breastfeeding, and cold virus and allergy symptoms. We later excluded data for two women because of their failure to rate one of the five odors. Statistical analysis was performed on data from 120 participants.

\subsection{Ethical Considerations}

This data was collected about the 2005. The project committee was approved by the Chang Gung University of Science and Technology (CGUST). The study complies with all regulations and confirmation that informed consent was obtained. The researcher has been de-identified the data for this study prior to analysis in accordance with the standards by the privacy rule for protecting personal health information. Hence, the data were analyzed and reported anonymously. To create a caring and friendly environment, each subject was known and allowed to discontinue without any reason in the experiment events. Confidentiality was maintained by data coding and statistical analysis was completed in 2013 accordingly.

\subsection{Dysmenorrheal Assessment}

A brief self-rating questionnaire for dysmenorrheal (DQ) experience was developed. The DQ was self-reported, with each person recording her pain-related experiences for the last year. The DQ included 4 sub-items (severity, frequency, duration, and strength). The DQ-severity and DQ-strength were given a weighted score of 1 to 10 and the DQ-duration was 6 points with higher scores indicating higher levels of pain and reflecting poor DE. Rating for the frequency subitem ranged from 1 to 5 points, with a higher score indicating lower pain levels in DE. 


\subsection{Odorants Material}

Five subsets of odors (lemon, $\mathrm{H}_{2} \mathrm{~S}$, ethyl, acetone, and rose) were used in the experiments. A visual analogue scale (VAS) was presented to participants to evaluate the intensity (strong to weak) and hedonic value (unpleasantness to pleasantness) of each odor. Odor intensity (arousal) was measured according to 6 levels, ranging from a score of 1 for extremely strong to 6 for not strong at all. The hedonic values measurement ranged from 1 for most unpleasant to 5 for most pleasant.

\subsection{Psychometric Tests}

Each participant completed self-reported psychological assessment after the odorant tests: (1) the Generalized Anxiety Disorder (GAD-10): GAD-10 is a 10 -item selfreport inventory, a weighted score of 0 to 4 . A score of 15 19, 20-29 and 30-50 points indicates a mild, moderate and severe condition of anxiety, respectively. It measures generalized anxiety according to severity scales by scoring the simple total sum of the items, the total score ranging from $0-50$; (2) the CES Depression Inventory (Center for Epidemiologic Studies Depression Scale; DI), which contained 20 items. Each item received a weighted score of 0 to 3 , with a rating of 3 indicating the highest level of depression. The total score range was 0 to $60 ;(3)$ short form survey of the Life Stress Level (SL) on a scale from 0 (not at all) to 10 (very strong).

\subsection{Statistical Analysis}

We used SPSS version 16.0 (SPSS Inc, Chicago, Illinois) performing statistical analyses. Descriptive statistical analyses of group characteristics and DQ sub-item (severity, strength, duration, and frequency) and psychometric tests (GAD-10, DI, SL) scores were conducted. Correlation analyses were performed to evaluate the association between DE variations, five odors perception and the psychometric testing scores. The inter-correlates for odor perception (intensity and hedonic value), DQ sub-item and psychometric properties of the GAD10, DI, SL scores were analyzed using Pearson's statistics. Analyses were performed separately for each of the 6 levels of intensity and 5 hedonic values of odorants.

After completing this simple correlation module for odorant perception and DQ index, we examined the best estimate factor for three DQ variables (severity, strength, frequency) to $\mathrm{H}_{2} \mathrm{~S}$ odor hedonic values by stepwise linear regression analysis. Because no significant association was observed between DQ-duration and the $\mathrm{H}_{2} \mathrm{~S}$ odor hedonic values, duration was excluded from the subsequent regression analyses. In all statistical tests, a $P$-value of $<.05$ (two-tailed) was considered significant.

\section{Results}

\subsection{Descriptive Data}

Table 1 presents the characteristics of the 120 participants including the DQ index, odor hedonic and intensity evaluation scores, and Psychometric properties, Mean value and standard deviation (mean $\pm \mathrm{SD}$ ) of the DQ scores for severity, duration, strength and frequency were $4.91 \pm 2.51$, $2.57 \pm 1.29,4.53 \pm 2.61$, and $2.64 \pm 1.25$, respectively. Table 1 presents the levels of intensity and hedonic values (mean \pm $\mathrm{SD}$ ) of the five odorants - lemon, rose, ethyl, acetone and $\mathrm{H}_{2} \mathrm{~S}$. The participants rated $\mathrm{H}_{2} \mathrm{~S}$ odorant as the most unpleasant (lowest valence mean), and the said odorant also received the strongest intensity among five odorants (Table 1). The lemon odorant was rated as the most pleasant and as having the lowest intensity compared to the other odorants. Mean value and standard deviation (mean $\pm \mathrm{SD}$ ) of the Psychometric test for GAD-10, DI, SL were 9.05 \pm 7.92 , $22.91 \pm 7.88$, and $6.20 \pm 1.98$ separately.

Table 1. Means and standard deviations of the DEQ scores, the intensity and hedonic values of the five odorants and Psychometric tests $(n=120)$.

\begin{tabular}{ll}
\hline & Mean \pm SD \\
\hline Dysmenorrhea Assessment & \\
DQ-severity & $4.91 \pm 2.51$ \\
DQ-duration & $2.57 \pm 1.29$ \\
DQ-strength & $4.53 \pm 2.61$ \\
DQ-frequency & $2.64 \pm 1.25$ \\
Odorant (Valence) & \\
Lemon & $3.30 \pm 0.79$ \\
Rose & $2.76 \pm 0.71$ \\
Ethyl & $2.48 \pm 0.86$ \\
Aceton & $2.54 \pm 0.67$ \\
$\mathrm{H}_{2} \mathrm{~S}$ & $1.61 \pm 0.71$ \\
Odorant (Intensity) & \\
Lemon & $4.13 \pm 1.07$ \\
Rose & $3.48 \pm 1.18$ \\
Ethyl & $2.52 \pm 1.14$ \\
Aceton & $3.03 \pm 1.24$ \\
$\mathrm{H}_{2} \mathrm{~S}$ & $2.04 \pm 1.17$ \\
Psychometric tests & \\
GAD-10 & \\
DI & b \\
SL & $9.05 \pm 7.29$ \\
\hline
\end{tabular}

Odorant intensity: 1 for extremely strong to 6 for not strong at all. The hedonic Values ranged 1 for most unpleasant to 5 for most pleasant.

* Plus-minus value are means $\pm \mathrm{SD}$.

a. GAD-10 measures generalized anxiety severity scales. It developed from the Hamilton 6-item anxiety scale (HAM-A6), the total score ranging from $0-50$, with higher scores indicating worse functional status.

b. The DI is a screening test for depression and depressive disorder. It measures symptoms defined by the American Psychiatric Association' Diagnostic and Statistical Manual (DSM-IV) for a major depressive episode.

c. The SL is a brief report of the subjects' reaction to overall life stress level, not limit to pain.

\subsection{Odor Perception and Dysmenorrhea}

We observed a strong link between DQ score (severity, strength, and frequency) and $\mathrm{H}_{2} \mathrm{~S}$ emotion valence among five odorants. Table 2 revealed that the unpleasant odorant $\mathrm{H}_{2} \mathrm{~S}$ rating was particularly associated with the DQ's severity, strength, and frequency scores $(P<.05)$. It indicated chemical 
sensitivity increasing of the $\mathrm{H}_{2} \mathrm{~S}$ odorant significant correlation with the degree of dysmenorrhea. (2) The lemon, acetone, and ethyl odorant were generally recognized as pleasant and the hedonic values did not remarkable results related to the DQ- sub-item pain index. (3) In Additional, perceptual intensity of the rose odorant was related to dysmenorrheal frequency levels $(P=.015)$ (Table2; upper column). Due to the DQ frequency has a reverse scoring scale, results showed the decreasing perception of rose intensity as predicted by women's dysmenorrhea frequency increased. Altogether, both odorant with increasing $\mathrm{H}_{2} \mathrm{~S}$ hedonic value and the rose intensity decline denoted significant relationships to DQ scores. Results indicate that chemical sensitivity changes of the perception of selected odors as functional predictors of negative DE.

Table 2. Correlations (Pearson's r) among the odor perception and dysmenorrheal experience (upper), psychometric tests. ( $n=120$ ).

\begin{tabular}{|c|c|c|c|c|c|c|c|c|c|c|}
\hline \multirow{2}{*}{$\begin{array}{l}\text { Odorant } \\
\text { Emotion }\end{array}$} & \multicolumn{2}{|l|}{ Lemon } & \multicolumn{2}{|l|}{ Rose } & \multicolumn{2}{|l|}{ Ethyl } & \multicolumn{2}{|l|}{ Aceton } & \multicolumn{2}{|l|}{$\mathbf{H}_{2} \mathrm{~S}$} \\
\hline & Valence & intensity & Valence & intensity & Valence & intensity & Valence & intensity & Valence & intensity \\
\hline \multicolumn{11}{|l|}{ Questionnaires } \\
\hline DEQ-severty & $p=.628$ & $p=.881$ & $p=.673$ & $p=.163$ & $p=.780$ & $p=.565$ & $p=.106$ & $p=.214$ & $p=.006^{* *}$ & $p=.331$ \\
\hline \multirow[t]{2}{*}{ DEQ-duration } & $r=.010$ & $r=-.036$ & $r=-.057$ & $r=-.107$ & $r=-.077$ & $r=-.006$ & $r=-.107$ & $r=-.041$ & $r=-.133$ & $r=.007$ \\
\hline & $p=.913$ & $p=.694$ & $p=.536$ & $p=.244$ & $p=.403$ & $p=.944$ & $p=.245$ & $p=.654$ & $p=.149$ & $p=.938$ \\
\hline \multirow[t]{2}{*}{ DEQ-strength } & $r=.025$ & $r=-.037$ & $r=.037$ & $r=-.101$ & $r=.050$ & $r=-.013$ & $r=-.086$ & $r=-.102$ & $r=-.239$ & $r=-.105$ \\
\hline & $p=.790$ & $p=.688$ & $p=.688$ & $p=.271$ & $p=.585$ & $p=.891$ & $p=.350$ & $p=.268$ & $p=.009 * *$ & $p=.252$ \\
\hline \multirow[t]{2}{*}{ DEQ-freq. } & $r=-.060$ & $r=-.059$ & $r=.063$ & $r=.222$ & $r=-.025$ & $r=.054$ & $r=.093$ & $r=.164$ & $r=.220$ & $r=.057$ \\
\hline & $p=.512$ & $p=.524$ & $p=.494$ & $p=.015^{*}$ & $p=.784$ & $p=.555$ & $p=.310$ & $p=.073$ & $p=.016^{*}$ & $p=.539$ \\
\hline \multicolumn{11}{|c|}{ Psychometric test } \\
\hline SAI & $r=-.050$ & $r=-.073$ & $r=.207$ & $r=.065$ & $r=.048$ & $r=-.049$ & $r=-.026$ & $r=.048$ & $r=-.096$ & $r=-.052$ \\
\hline \multirow[t]{2}{*}{ DI } & $r=-.151$ & $r=-.028$ & $r=.119$ & $r=.078$ & $r=.074$ & $r=-.058$ & $r=-.170$ & $r=-.074$ & $r=-.179$ & $r=-.152$ \\
\hline & $p=.101$ & $p=.763$ & $p=.194$ & $p=.400$ & $p=.421$ & $p=.532$ & $p=.063$ & $p=.424$ & $p=.050^{*}$ & $p=.098$ \\
\hline \multirow[t]{2}{*}{ SL } & $r=-.110$ & $r=-.083$ & $r=.085$ & $r=-.131$ & $r=.041$ & $r=-.020$ & $r=-.037$ & $r=-.002$ & $r=.216$ & $r=-.180$ \\
\hline & $p=.233$ & $p=.368$ & $p=.354$ & $p=.153$ & $p=.655$ & $p=.830$ & $p=.687$ & $p=.984$ & $p=.018^{*}$ & $p=.049 *$ \\
\hline
\end{tabular}

$*$ =significant at the level of $0.05 ; * *=$ significant at the level of 0.01

Changes in perceived hedonic values of the $\mathrm{H}_{2} \mathrm{~S}$ odorant associated with the DQ scores for severity, frequency, and strength level $(P<.05)$. Decreased perception of the intensity of the rose odorant related to predict dysmenorrheal frequency levels $(P<.05)$ (DQ frequency has a reverse scoring scale). DQ: dysmenorrhea questionnaire; GAD: generalize anxiety disorder; DI: depression inventory; SL: life stress level.

\subsection{Odor Perception and Psychometric Tests}

Independent measurements of the life stress levels were significantly related to the intensity of the $\mathrm{H}_{2} \mathrm{~S}$ odorant and hedonic level $(P<.05)$ (Table 2, lower column). The hedonic level of the $\mathrm{H}_{2} \mathrm{~S}$ odorant corresponded to a significant relation for DI $(P=.05)$. The hedonic level of the rose odorant also represented a significant predictive variable for anxiety $(P<.05)$. There were no significant relationships among the hedonic values/intensities of the lemon, acetone and ethyl odorants and GAD-10, DI and SL scores (Table 2, lower column)

\subsection{Regression Analysis for DQ Index and $\mathrm{H}_{2} \mathrm{~S}$ Hedonic Values}

After obtaining the correlation results of the $\mathrm{H}_{2} \mathrm{~S}$ hedonic values, and among DQ severity, frequency, and rating, we observed that collinearity existed between the three DQ variables (Table 3). DQ-severity score was the statistical significance of the predictor variables on $\mathrm{H}_{2} \mathrm{~S}$ odorant perception alteration $(P=.006)$ by stepwise regression analysis. DQ-frequency and rating were excluded by model of that $p$-value $>0.05$ (Table 4$)$.
Table 3. Correlations (Pearson's r) among DQ- severity, frequency and rating.

\begin{tabular}{llll}
\hline & & severity & frequency \\
\hline frequency & Correlation & -0.617 & \\
& $P$-Value & 0.000 & \\
rating & Correlation & 0.844 & -0.598 \\
& $P$-Value & 0.000 & 0.000 \\
\hline
\end{tabular}

Three independent variables exhibited collinearity and significant linear relationships between each other $(p<0.05)$.

Table 4. Results of Stepwise Regression Analysis predicting H2S odor values $(n=120)$.

\begin{tabular}{lllll}
\hline hedonic Predictor & Beta & SE Coef & T & $\boldsymbol{P}$ \\
\hline Constant & 1.951 & 0.139 & 14.07 & 0.000 \\
severity & -0.070 & 0.025 & -2.780 & 0.006 \\
Excluded Variables & Beta in & Partial Correlation & $\mathrm{T}$ & $P$ \\
frequency & .106 & 0.086 & 0.930 & 0.354 \\
rating & -.103 & -.057 & -0.615 & 0.540 \\
\hline
\end{tabular}

DQ -severity score was the statistical significance of the predictor variables on $\mathrm{H}_{2} \mathrm{~S}$ odorant perception alteration. DQ-frequency and rating were excluded by model of that $p$-value $>0.05$. 


\subsection{Psychometric Tests and Dysmenorrhea Experience}

Pearson's correlations indicate that a negative DE (4 DQ scores sub-items) was associated with increased psychological distress (i.e., the GAD-10, DI, SL, and DQ scores). There was a significant correlation between the GAD-10 score and the DQ scores [severity, strength $(P$ $=.000)$; frequency $(P<.05)]$. The DI score positively related to the DQ scores for severity $(P<.005)$ and strength $(P$ $=.000)$. The SL score was significantly correlated to the $4 \mathrm{DQ}$ sub-item scores [severity, strength $(P=.000)$ and duration, frequency $(P<.05)$

\section{Discussion}

No prior study has investigated the long-term effects of dysmenorrhea on odor perception. The aim of the current study was to test the hypothesis of potential variations in chemical sensitivity in dysmenorrheal women. Results demonstrated that changes in perception of selected odors, specifically the increasing unpleasantness of the $\mathrm{H}_{2} \mathrm{~S}$ odorant and weakening intensity of the rose odorant, were associated with poor DE. Higher levels of psychological distress, as indicated by higher GAD-10, DI and SL scores, were significantly related to higher DQ sub-item scores. The observed olfactory sensitivity changes may reflect olfactorylimbic dysfunction or damage in dysmenorrhea women with poor control. Findings suggest the need for appropriate management of menstrual cramps in women and health.

Unpleasantness odor $\mathrm{H}_{2} \mathrm{~S}$ perception change in dysmenorrhea women

In this study, we observed a significant correlation between pain index scores and perception of selected odors. Unpleasantness ratings for the $\mathrm{H}_{2} \mathrm{~S}$ odorant significantly related to poor DE. Increasing negative responses to $\mathrm{H}_{2} \mathrm{~S}$ were significantly related to DQ severity, strength, and frequency $(P<.05)$. The DQ- severity was a statistically significant predictor of $\mathrm{H}_{2} \mathrm{~S}$-related hedonic values $(P=.006)$, after controlling the collinear variables (Table 4). Moreover, we found that the decrease in perceived intensity of the rose odorant showed a significant correlation with the DQ frequency score $(P=.015)$. Previous studies have described the association between functional changes in the olfactorylimbic areas and chronic pain. These regions included the anterior olfactory nucleus, periamygdaloid and orbitofrontal cortex areas that coded for emotional hedonic value/intensity in the chemosensory domain [12-15]. Dysmenorrhea is accompanied by abnormal brain activity and morphological changes in several brain regions, which neurocircuits are involved in various aspects of affective-pain processing [1, 2]. The circuitry of the olfactory system is located in the basal limbic forebrain[16]. Converging evidence has also suggested that the amygdala, which is critically involved in sensitivity to valence stimuli and arousal [17], responds to aversive olfactory stimulation [15]. The morphology of limbic-amygdala circuitry changes in PDM [2] could explain our observations that the trend of the olfactory perception changes following women's dysmenorrhea score. Further, the manifestation of decreasing positive response to the rose odorant and an increasing aversive response to the $\mathrm{H} 2 \mathrm{~S}$ odorant would debate on afterwards.

Rose odorant and $\mathrm{H}_{2} \mathrm{~S}$ perception sensitivity change in dysmenorrhea women

The rose odorant perceptual intensity was negative associated with the high DQ frequency group as described previously (Table2, upper column). The participants generally rated the $\mathrm{H}_{2} \mathrm{~S}$ odor as an unpleasant/ aversive chemical signal and the rose odor as a pleasant chemical signal (Table 1) [18]. Contrasting exhibition of increasing unpleasant emotion of $\mathrm{H}_{2} \mathrm{~S}$ odor and weakening intensity to the pleasant rose odor in dysmenorrheal women is perhaps behind evolutional manners. The desire to experience more pleasure and to avoid pain is fundamental human behaviors [19]. The results of our study show that the reverse of such behavior could occur in women with poor dysmenorrhea experience as shown by the reaction to weakening rose intensity and increasing $\mathrm{H}_{2} \mathrm{~S}$ unpleasantness [12]. Studies have suggested that the olfactory system plays a role as a sentinel system rather than a fine-tuned selection system [20]. As such, its central function would be to produce emotional responses to odors that would serve as the base for approachavoidance behaviors [21]. Labroo and Nielsen (2010) suggested that people can subconsciously reverse the approach-avoidance relationship [13], describing the possibility of reducing aversion to certain immediately aversive items that may be beneficial to health. Internal mechanisms have evolved in both humans and animals, enabling them to maximize their chances of survival when encountering threats, by responding to adverse information that indicates risk and very quickly determining whether the environmental stimulus is dangerous or not. Conversely, weakening the intensity value of the pleasantness of the rose odorant reflects the potential necessity to heighten rose odorant application in the high DQ frequency group. It remains to be elucidated if this functional resilience results in improved adaptive responses for dysmenorrhea women.

Dysmenorrhea experience and it's associated with Psychometric Factors

There is a strong link between DQ score and psychological distress such as anxiety, depression and stress level in our findings. Consistent with previous studies, the four DQ subitems were significantly correlated with individual psychometric properties of the scores (GAD-10, DI, SL)[4, 22, 23]. Previous studies have suggested that relatively high psychiatric comorbidity, stressful personal histories and psychological properties are predictive indicators in clinicbased chronic pain patients [24, 25]. Wang et al also linked the incidence of dysmenorrhea to stress [23], providing further evidence to suggest that chronic pain is a common comorbidity of mood and anxiety disorders [22]. Results perceive that the bio-psychological interrelation may be a prolific area to explore for future investigations dysmenorrhea.

Psychometric property related odorant perception 


\section{Anxiety}

Moreover, an interesting result emerged is showing in our correlation analysis. The hedonic value of the rose odorant represented a predictive variable for anxiety $(P=.024)$ (Table 2; lower column). The present study identified a significant relationship between GAD-10 scores and emotional response to the rose odorant $(P<.05)$ (Table 3$)$. This indicated that participants with higher anxiety assigned higher values for pleasantness to the rose odorant. In 2007, Bradley et al. described the association of rose oil with anxiolytic effects [26]. However, the underlying mechanism remains unclear. Rose oil is a complex mixture of chemicals that acts on multiple pathways. In a series of studies conducted by Gureje et al, people with repeated pains displayed susceptibility to psychological disorders such as depression and anxiety [25, 27]. The increasing pleasantness of the rose odorant is associated with the high DQ frequency group as described previously. The results of our study can further indicate that potential use of aromatic treatments may have a relieving effect on physio-psychological sorrow and may be beneficial to women and health.

\section{Life Stress level}

The associations of life stress level and five odorant perceptions were also assessed in the present study. Findings were related to previous studies which described the potential roles of stress, in development and expression of the multiple chemicals sensitivity in the participants under restraint stress, fatigue or chronic illness [19, 28, 29]. Results showed that a significant correlation between the intensity and hedonic value of the $\mathrm{H}_{2} \mathrm{~S}$ odorant and SL score. Stress could produce variety of behaviors in animals and humans [29] and chronic stress leads to changes in other neural circuits, such as the olfactory system also documented [30]. One major target of the hippocampus, which belongs to limbic system, is sensitivity to chronic stress [19, 28, 31]. Stress activates the hypothalamic-pituitary-- adrenal (HPA) axis, altering morphology and function of the amygdala- hippocampal regions [14]. The amygdala is critically involved in emotional arousal, interacting with stress hormones and increasing perception of the emotional valence of events $[17,32]$. Chronic stress causes dysfunctions in other brain regions as well, especially the limbic system that is responsible for emotive regulation [3336]. In the present study, the significant relationships between the increased SL scores and elevated $\mathrm{H}_{2} \mathrm{~S}$ odor sensitivity might reflect warning signals that lead to avoidance behavior and minimizing injury for an organism.

Clinical relevance of the findings

Finding elucidated a significant association between increase chemical sensitivity and self-report DQ pain index. Change of odor thresholds highlight a lead that olfactory sensitization development probably through adverse dysmenorrhea. Chemical Sensitivity is the beginning of a syndrome that can progress to olfactory sensitization, neurological disease, autoimmunity and many other health problems [37-40]. From prior knowledge, most people often tolerate well, endure or ignore their smell capability. Perhaps, they had not yet appeared serious olfactory symptoms or sensation variations; hence do not seek medical evaluation or treatment. However, exposure to environmental odors could have a negative impact on health. Increased sensitivity to adverse chemicals in the environment may further induce top-down hurt. The risk of having dysmenorrhea is highest among women experiencing high stress and negative emotions. The findings regarding the significant chemical sensory response, strengthen the risk of neural sensitization occurring predominantly in poorly controlled dysmenorrhea. Chronic illness could provoke Multiple Chemical Sensitivities (MCS) referred to as Idiopathic Environmental Intolerance [41]. The results of this study provide a new impetus to the development of preventive medicine or preventive care strategies to minimize the risk of chemical sensitization by managing menstrual cramps.

\section{Conclusion}

This is the first study to report the long-term effects of dysmenorrhea on odor perception. Findings indicate that changes in perceived hedonic values of the $\mathrm{H}_{2} \mathrm{~S}$ odorant and in intensity of the rose odorant represent predictive variables for poor dysmenorrheal experience. These chemical sensitivity changes may reflect olfactory-limbic dysfunction in the women experiencing severe dysmenorrheal symptoms. The psychological distress and somatic sensitization pose a potential risk to dysmenorrheic women. Our results highlight the chemosensory evaluations and appropriate management of menstrual cramps is an important requirement in women's health.

\section{Acknowledgements}

We thank the 120 participants from the Chang Gung University of Science and Technology (CGUST), as well as CGUST for providing administrative support. The authors would like to thank Lee-Fen $\mathrm{Ni}$ for statistical counseling. In addition, we would like to thank IBRU of our front line colleagues for their consultation. No potential conflict of interest relevant to this article is reported. Data was collected in 2005 and completely analyzed in 2013. The funding of study was sponsored by grant from Ministry of Science and Technology Taiwan (NSC 101-2629-B-255 -001 -MY2) and Chang Gung Memorial Hospital (BMRP C52), supply for statistic consult, document editing and successive publication. The authors declare that they have no competing interests. We also sincerely appreciate the editor's patience in reviewing this article.

\section{References}

[1] Tu CH, et al.: Abnormal cerebral metabolism during menstrual pain in primary dysmenorrhea. Neuroimage 2009, 47(1): p. $28-35$

[2] $\mathrm{Tu} \mathrm{CH}$, et al.: Brain morphological changes associated with cyclic menstrual pain. Pain 2010, 150(3): p. 462-468. 
[3] Bajaj P, H. Madsen, and L. Arendt-Nielsen: A comparison of modality-specific somatosensory changes during menstruation in dysmenorrheic and nondysmenorrheic women. Clin J Pain 2002, 18(3): p. 180-190.

[4] Granot $M$, et al.: Pain perception in women with dysmenorrhea. Obstet Gynecol 2001, 98(3):p. 407-411.

[5] Stein MB, et al.: Increased amygdala and insula activation during emotion processing in anxiety-prone subjects. Am $J$ Psychiatry 2007, 164(2):p. 318-327.

[6] Cantor C: Post-traumatic stress disorder: evolutionary perspectives. Aust N Z J Psychiatry 2009, 43(11): p. 10381048.

[7] Onur OA, et al: Noradrenergic enhancement of amygdala responses to fear. Soc Cogn Affect Neurosci 2009, 4(2): p. 119-126.

[8] Golub ET, Latka M, Hagan H, Havens JR, Hudson SM, Kapadia F, Campbell JV, Garfein RS, Thomas DL, Strathdee $\mathrm{SA}$ et al: Screening for depressive symptoms among HCVinfected injection drug users: examination of the utility of the CES-D and the Beck Depression Inventory. Journal of urban health: bulletin of the New York Academy of Medicine 2004, 81(2):278-290.

[9] Okun A, Stein RE, Bauman LJ, Silver EJ: Content validity of the Psychiatric Symptom Index, CES-depression Scale, and State-Trait Anxiety Inventory from the perspective of DSMIV. Psychological reports 1996, 79(3 Pt 1):1059-1069.

[10] Waehrens EE, Amris K, Bartels EM, Christensen R, Danneskiold-Samsoe B, Bliddal H, Gudbergsen H: Agreement between touch-screen and paper-based patient-reported outcomes for patients with fibromyalgia: a randomized crossover reproducibility study. Scandinavian journal of rheumatology 2015:1-8.

[11] David AG, Kenneth, F. S.: Epidemiology series-Descriptive studies: what they can and cannot do. LANCET 2002, 359 (12):p. 145-149.

[12] Higgins ET: Beyond pleasure and pain. Am Psychol 1997, 52(12):p. $1280-1300$.

[13] Nielsen AALaJH: Half the Thrill Is in the Chase: Twisted Inferences from Embodied Cognitions and Brand Evaluation. Journal of Consumer Research 2010, 37(1):p. 143-158.

[14] Ulrich-Lai YMaJPH: Neural regulation of endocrine and autonomic stress responses. Nat Rev Neurosci 2009, 10(6):p. 397-409.

[15] Zald DHaJV: Pardo, Emotion, olfaction, and the human amygdala: amygdala activation during aversive olfactory stimulation. Proceedings of the National Academy of Sciences of the United States of America 1997, 94(8):p. 4119-4124.

[16] Gottfried JA, et al.: Functional heterogeneity in human olfactory cortex: an event-related functional magnetic resonance imaging study. $J$ Neurosci 2002, 22(24): p. 1081910828.

[17] Reilly SaMAB: Conditioned taste aversion and amygdala lesions in the rat: a critical review. Neurosci Biobehav Rev 2005, 29(7):p. 1067-1088.

[18] Seubert J, Gregory KM, Chamberland J, Dessirier JM, Lundstrom JN: Odor valence linearly modulates attractiveness, but not age assessment, of invariant facial features in a memory-based rating task. PloS one 2014, 9(5):e98347.

[19] Fiedler N, Kelly-McNeil K, Ohman-Strickland P, Zhang J, Ottenweller J, Kipen HM: Negative affect and chemical intolerance as risk factors for building-related symptoms: a controlled exposure study. Psychosom Med 2008, 70(2):254262.

[20] Fjaeldstad A: Testing olfactory function and mapping the structural olfactory networks in the brain. Dan Med J 2018, 65(1).

[21] Lundstrom JN, Seven S, Olsson MJ, Schaal B, Hummel T: Olfactory event-related potentials reflect individual differences in odor valence perception. Chemical senses 2006, 31(8):705-711.

[22] Gureje $\mathrm{O}$, et al.: The relation between multiple pains and mental disorders: results from the World Mental Health Surveys. Pain 2008, 135(1-2):p. 82-91.

[23] Wang L, et al.: Stress and dysmenorrhoea: a population based prospective study. Occupational and environmental medicine 2004, 61(12):p. 1021-1026.

[24] Currie SRaJW: Chronic back pain and major depression in the general Canadian population. Pain 2004, 107(1-2):p. 54-60.

[25] Gureje O: Comorbidity of pain and anxiety disorders. Curr Psychiatry Rep 2008, 10(4):p. 318-322.

[26] Bradley BF, et al.: The effects of prolonged rose odor inhalation in two animal models of anxiety. Physiol Behavior 2007, 92(5):p. 931-938.

[27] Gureje O: Treating chronic pain in the context of comorbid depression. Pain 2008, 134(1-2):p. 3-4.

[28] Bell IR, et al.: Individual differences in neural sensitization and the role of context in illness from low-level environmental chemical exposures. Environ Health Perspect 1997, 105 Suppl 2:p. 457-466.

[29] Andersson L, Claeson AS, Nyberg L, Nordin S: Short-term olfactory sensitization involves brain networks relevant for pain, and indicates chemical intolerance. Int $\mathrm{J}$ Hyg Environ Health 2017, 220(2 Pt B):503-509.

[30] Yuan TF, Hou G, Arias-Carrion O: Chronic stress impacts on olfactory system. CNS \& neurological disorders drug targets 2015, 14(4):486-491.

[31] Eisch AJ, Petrik D: Depression and hippocampal neurogenesis: a road to remission? Science 2012, 338(6103):72-75.

[32] Tottenham NaMAS: A review of adversity, the amygdala and the hippocampus: a consideration of developmental timing. Front Hum Neurosci 2009, 3:p. 68.

[33] Bielau H, Brisch R, Gos T, Dobrowolny H, Baumann B, Mawrin C, Kreutzmann P, Bernstein HG, Bogerts B, Steiner J: Volumetric analysis of the hypothalamus, amygdala and hippocampus in non-suicidal and suicidal mood disorder patients--a post-mortem study. CNS \& neurological disorders drug targets 2013, 12(7):914-920.

[34] Hou G, Tian R, Li J, Yuan TF: Chronic stress and Parkinson's disease. CNS neuroscience \& therapeutics 2014, 20(1):1-2. 
[35] Hou G, Xiong W, Wang M, Chen X, Yuan TF: Chronic stress influences sexual motivation and causes damage to testicular cells in male rats. The journal of sexual medicine 2014, 11(3):653-663.

[36] Yuan TF, Hou G: The effects of stress on glutamatergic transmission in the brain. Molecular neurobiology 2015, 51(3):1139-1143.

[37] Graveling RA, Pilkington A, George JP, Butler MP, Tannahill $\mathrm{SN}$ : A review of multiple chemical sensitivity. Occupational and environmental medicine 1999, 56(2):73-85.

[38] Labarge XS, McCaffrey RJ: Multiple chemical sensitivity: a review of the theoretical and research literature. Neuropsychology review 2000, 10(4):183-211.
[39] Mozhaev EA, Golubev IR: [Multiple chemical sensitivity syndrome. Review]. Gigiena i sanitariia 2000(6):48-50.

[40] Nowak D, Pedrosa Gil F, Angerer P, Tretter F, Eis D: [Multiple chemical sensitivity (MCS): a review]. Deutsche medizinische Wochenschrift 2005, 130(47):2713-2718.

[41] Hawkins JL, Moore NJ, Miley D, Durham PL: Secondary traumatic stress increases expression of proteins implicated in peripheral and central sensitization of trigeminal neurons. Brain Res 2018, 1687:162-172. 